\title{
Kybernetika
}

\section{Hideki Sano}

On approximation of stability radius for an infinite-dimensional feedback control system

Kybernetika, Vol. 52 (2016), No. 5, 824-835

Persistent URL: http://dml.cz/dmlcz/145970

\section{Terms of use:}

(C) Institute of Information Theory and Automation AS CR, 2016

Institute of Mathematics of the Czech Academy of Sciences provides access to digitized documents strictly for personal use. Each copy of any part of this document must contain these Terms of use.

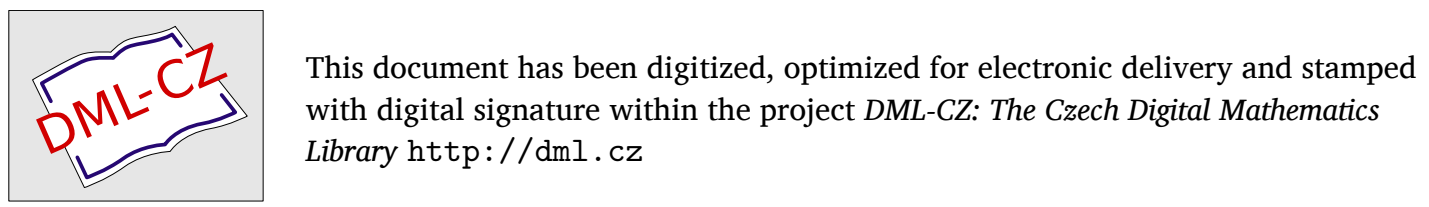




\title{
ON APPROXIMATION OF STABILITY RADIUS FOR AN INFINITE-DIMENSIONAL FEEDBACK CONTROL SYSTEM
}

\author{
HIDEKI SANO
}

In this paper, we discuss the problem of approximating stability radius appearing in the design procedure of finite-dimensional stabilizing controllers for an infinite-dimensional dynamical system. The calculation of stability radius needs the value of $H_{\infty}$-norm of a transfer function whose realization is described by infinite-dimensional operators in a Hilbert space. From the computational point of view, we need to prepare a family of approximate finite-dimensional operators and then to calculate the $H_{\infty}$-norm of their transfer functions. However, it is not assured that they converge to the value of $H_{\infty}$-norm of the original transfer function. The purpose of this study is to justify the convergence. In a numerical example, we treat parabolic distributed parameter systems with distributed control and distributed/boundary observation.

Keywords: distributed parameter system, finite-dimensional controller, stability radius, transfer function, semigroup

Classification: 93D15, 93C25

\section{INTRODUCTION}

In the field of control of distributed parameter systems, the linear system described by the following evolution equation with output equation has been used for a long time.

$$
\begin{aligned}
\dot{z}(t) & =-A z(t)+B u(t), \quad t>0, \quad z(0)=z_{0}, \\
y(t) & =C z(t), \quad t>0,
\end{aligned}
$$

where $-A$ is the infinitesimal generator of a $C_{0}$-semigroup on a real Hilbert space $H$ with inner product $\langle\cdot, \cdot\rangle$ and norm $\|\cdot\| . B: \mathbf{R}^{m} \rightarrow H$ is a bounded input operator, and $C: D(C) \subset H \rightarrow \mathbf{R}^{p}$ is a bounded/unbounded output operator. $z(t) \in H$ is the state variable, $u(t) \in \mathbf{R}^{m}$ the input variable, and $y(t) \in \mathbf{R}^{p}$ the output variable. For system (1), (2), the stabilization problem/the optimal control problem by static controllers have been investigated by many researchers (see e.g. [4, 7] and the references therein). Also, the stabilization problem by finite-dimensional dynamic controllers has been widely studied. In the following, we briefly survey several works related to the latter stabilization problem. In this paper, we especially treat a problem that remains in the design method based on stability radius.

DOI: $10.14736 /$ kyb-2016-5-0824 
In general, when one constructs a finite-dimensional model for an infinite-dimensional system and applies a finite-dimensional controller designed for it to the original infinitedimensional system, spillover phenomenon may be occured by the influence of unmodeled modes. Sakawa firstly introduced two kinds of finite-dimensional observers for linear diffusion systems to reduce the influence of unmodeled modes for the closed-loop system with the finite-dimensional controller [12. After that, Balas called one of them as the residual mode filter (RMF), and clarified that the RMF plays an essential role for the construction of finite-dimensional stabilizing controllers [1]. Moreover, it was shown in 13. that the results could be extended to the system with bounded input operator and $A^{\gamma}$-bounded output operator. On the other hand, Nambu gave the design method of infinite-dimensional stabilizing controllers applicable to linear parabolic systems under boundary control and observation, and further accomplished finite-dimensionalization of the obtained controllers [8]. Schumacher gave the direct design method of finitedimensional controllers for a wide class of linear time-invariant systems [16, in which the eigenfunctions of the operator $-A+B F$ were used. Moreover, Schumacher's design method was extended to linear parabolic systems with unbounded control and observation by Curtain [3]. Also, Lasiecka gave finite element approximation of Luenberger's observer based controllers for linear parabolic systems with unbounded input and output operators [7, Chapter 4]. Thus, the existence of finite-dimensional controllers was assured theoretically for the systems mentioned above, however, these desgin methods had a common weak point that one could not give the order of controllers a priori, that is, the order was supposed to be taken sufficiently large, except the design method based on stability radius by El Jai and Pritchard [5, Chapter 1].

Although the method based on stability radius [5, Chapter 1] is very simple, in order to calculate stability radius we need the value of $H_{\infty}$-norm of a transfer function whose realization is described by infinite-dimensional operators in a Hilbert space. From the computational point of view, we need to prepare a family of approximate finitedimensional operators and then to calculate the $H_{\infty}$-norm of their transfer functions. Then, we have a question of whether or not they converge to the value of $H_{\infty}$-norm of the original transfer function, which gives a motivation of this paper. The purpose of this study is to justify the convergence, that is, to show a theory of approximation for Theorem 1.1 below, and further to give a numerical example to illustrate the assertion. Here, we note that in [6] the method via numerical analysis has been proposed for approximation of stability radii for high order finite-dimensional systems.

To explain the existing result [5] briefly, we shall consider the case where the operator $C$ is bounded, i. e., $D(C)=H$, and the operator $A$ is defined by

$$
\begin{aligned}
& A f=\sum_{i=1}^{\infty} \lambda_{i}\left\langle f, \varphi_{i}\right\rangle \varphi_{i}, \quad f \in D(A), \\
& D(A)=\left\{f \in H ; \sum_{i=1}^{\infty} \lambda_{i}^{2}\left\langle f, \varphi_{i}\right\rangle^{2}<+\infty\right\},
\end{aligned}
$$

where $\left\{\lambda_{i}, i \geq 1\right\}$ is a sequence of real numbers such that $\lambda_{1}<\lambda_{2}<\cdots<\lambda_{i}<$ $\cdots, \lim _{i \rightarrow \infty} \lambda_{i}=\infty$, and $\left\{\varphi_{i}, i \geq 1\right\}$ forms a complete orthogonal system in $H$. It is clear that the operator $A$ is self-adjoint. Then, it follows from Hille-Yosida's theorem [9] 
that $-A$ generates a $C_{0}$-semigroup $e^{-t A}$ on $H$ whose expression is given by

$$
e^{-t A} f=\sum_{i=1}^{\infty} e^{-\lambda_{i} t}\left\langle f, \varphi_{i}\right\rangle \varphi_{i}, \quad t \geq 0, f \in H
$$

In order to decompose system (1), (2), we use the orthogonal projection $P_{k}$ defined by $P_{k} f=\sum_{i=1}^{k}\left\langle f, \varphi_{i}\right\rangle \varphi_{i}$. Using the operators $P_{l}$ and $I-P_{l}$, where $l$ is a positive integer, we decompose the state variable $z(t)$ as $z(t)=z_{1}(t)+z_{2}(t)$, where $z_{1}(t):=P_{l} z(t)$, $z_{2}(t):=\left(I-P_{l}\right) z(t)$. Also, the space $H$ is expressed as

$$
H=\overbrace{P_{l} H}^{\operatorname{dim}=l} \oplus \overbrace{\left(I-P_{l}\right) H}^{\operatorname{dim}=\infty} .
$$

Then, system (1), (2) is equivalently expressed as follows (see e.g. 1]):

$$
\begin{cases}\dot{z}_{1}(t)=-A_{1} z_{1}(t)+B_{1} u(t), & z_{1}(0)=z_{01} \\ \dot{z}_{2}(t)=-A_{2} z_{2}(t)+B_{2} u(t), & z_{2}(0)=z_{02} \\ y(t)=C_{1} z_{1}(t)+C_{2} z_{2}(t) & \end{cases}
$$

where

$$
\begin{array}{ll}
A_{1}:=P_{l} A P_{l}, & A_{2}:=\left(I-P_{l}\right) A\left(I-P_{l}\right), \\
B_{1}:=P_{l} B, & B_{2}:=\left(I-P_{l}\right) B, \\
C_{1}:=C P_{l}, & C_{2}:=C\left(I-P_{l}\right), \\
z_{01}:=P_{l} z_{0}, & z_{02}:=\left(I-P_{l}\right) z_{0} .
\end{array}
$$

In the above, note that the operator $A_{2}$ is unbounded, whereas all the other operators are bounded. Since the finite-dimensional Hilbert space $P_{l} H$ is identified with the Euclidean space $\mathbf{R}^{l}$ with respect to the basis $\left\{\varphi_{1}, \varphi_{2}, \ldots, \varphi_{l}\right\}$, each element in $P_{l} H$ is identified with an $l$-dimensional vector, and the operators $A_{1}, B_{1}$, and $C_{1}$ are identified with matrices with appropriate size.

\section{Assumption 1.}

(i) The integer $l$ is chosen such that the eigenvalues of the matrix $-A_{1}, \sigma\left(-A_{1}\right)$ contains all unstable eigenvalues of the operator $-A$.

(ii) The pair $\left(-A_{1}, B_{1}\right)$ is stabilizable and the pair $\left(C_{1},-A_{1}\right)$ is detectable (see e.g. [17. for the definitions and the related theorems).

Under (ii) of Assumption 1 , it is possible to choose a matrix $F_{1}$ such that $-A_{1}-B_{1} F_{1}$ is Hurwitz stable [17, since the pair $\left(-A_{1}, B_{1}\right)$ is stabilizable. Similarly, it is possible to choose a matrix $G_{1}$ such that $-A_{1}-G_{1} C_{1}$ is Hurwitz stable, since the pair $\left(C_{1},-A_{1}\right)$ is detectable. Here, let us consider the following observer-based controller [17] for system (1), (2):

$$
\left\{\begin{array}{l}
\dot{w}_{1}(t)=-A_{1} w_{1}(t)+B_{1} u(t)+G_{1}\left(y(t)-C_{1} w_{1}(t)\right), \quad w_{1}(0)=w_{10} \\
u(t)=-F_{1} w_{1}(t)
\end{array}\right.
$$


Then, by introducing the error vector $e_{1}(t)^{T}:=z_{1}(t)^{T}-w_{1}(t)^{T}$, the closed-loop system consisting of system (1), (2) and the controller (4) is written as

$$
\dot{\xi}(t)=(\mathcal{A}+\mathcal{B K} \mathcal{C}) \xi(t), \quad \xi(0)=\xi_{0},
$$

where the state $\xi(t):=\left[e_{1}(t)^{T}, z_{1}(t)^{T}, z_{2}(t)\right]^{T}$ is in the real Hilbert space $Z:=\mathbf{R}^{l} \times \mathbf{R}^{l} \times$ $\left(I-P_{l}\right) H$, and the operators $\mathcal{A}, \mathcal{B}, \mathcal{C}$, and $\mathcal{K}$ are defined by

$$
\begin{gathered}
\mathcal{A}=\left[\begin{array}{ccc}
-A_{1}-G_{1} C_{1} & 0 & 0 \\
B_{1} F_{1} & -A_{1}-B_{1} F_{1} & 0 \\
B_{2} F_{1} & -B_{2} F_{1} & -A_{2}
\end{array}\right], \\
\mathcal{B}=\left[\begin{array}{c}
-G_{1} \\
0 \\
0
\end{array}\right], \quad \mathcal{C}=\left[\begin{array}{lll}
0 & 0 & C_{2}
\end{array}\right], \quad \mathcal{K}=1 .
\end{gathered}
$$

Then, the following theorem is known.

Theorem 1.1. 5] Suppose that Assumption 1 is satisfied. Then, the operator $\mathcal{A}$ defined by (6) generates an exponentially stable $C_{0}$-semigroup $e^{t \mathcal{A}}$ on $Z$. In addition, if the condition

$$
\left\|\mathcal{C}(\cdot I-\mathcal{A})^{-1} \mathcal{B}\right\|_{\infty}:=\sup _{\omega \in \mathbf{R}}\left\|\mathcal{C}(j \omega I-\mathcal{A})^{-1} \mathcal{B}\right\|<1
$$

that is,

$$
\sup _{\omega \in \mathbf{R}}\left\|C_{2}\left(j \omega I+A_{2}\right)^{-1} B_{2} F_{1}\left(j \omega I+A_{1}+B_{1} F_{1}\right)^{-1}\left(j \omega I+A_{1}\right)\left(j \omega I+A_{1}+G_{1} C_{1}\right)^{-1} G_{1}\right\|<1
$$

is satisfied, the closed-loop operator $\mathcal{A}+\mathcal{B K C}$ of (5) also generates an exponentially stable $C_{0}$-semigroup $e^{t(\mathcal{A}+\mathcal{B K C})}$ on $Z$. In other words, the control law 4 becomes a finite-dimensional stabilizing controller for system (1), 22).

The proof of Theorem 1.1 is due to the result with respect to the stability radius 2, 11. As shown in [11, 2], the stability radius $r_{c}(\mathcal{A} ; \mathcal{B}, \mathcal{C})$ of the closed-loop system (5) is calculated as

$$
r_{c}(\mathcal{A} ; \mathcal{B}, \mathcal{C})=\frac{1}{\sup _{\omega \in \mathbf{R}}\|G(j \omega)\|}=\frac{1}{\|G(\cdot)\|_{\infty}},
$$

where $G(j \omega):=\mathcal{C}(j \omega I-\mathcal{A})^{-1} \mathcal{B}$. Therefore, when $r_{c}(\mathcal{A} ; \mathcal{B}, \mathcal{C})>\|\mathcal{K}\|=1$, that is, the condition (7) holds, the conclusion of the theorem immediately follows.

In Theorem 1.1, we note that the algorithm needs iteration of infinite times to check the condition (7), since it contains the infinite-dimensional operators $A_{2}, B_{2}$, and $C_{2}$. In Section 2, we discuss whether or not it is possible to approximate the operators $A_{2}$, $B_{2}$, and $C_{2}$ of the theorem by finite-dimensional operators. The novelty of this paper is the point that it proves the convergence of approximate stability radius to the original one in the feedback control system. Moreover, instead of (2), we discuss the case with unbounded output operator such as

$$
y(t)=\tilde{C}(A+c)^{\gamma} z(t), \quad 0<\gamma<1,
$$

where $A$ is the unbounded operator defined by $(3), \tilde{C}: H \rightarrow \mathbf{R}^{p}$ is a bounded linear operator, and $c$ is a constant chosen such that $\lambda_{1}+c>0$. 
Remark 1.2. In 10, the finite-dimensional version of this theorem was given. That is, the design method of low order stabilizing controllers was proposed for high order finite-dimensional systems, by using stability radius.

Remark 1.3. The condition (7) is a sufficient condition for spillover phenomenon not to be occured for the feedback control system consisting of system (1), (2) and the control law (4).

\section{MAIN RESULT}

By using the orthogonal projection $P_{k}$ defined in Section 1, we decompose the state variable $z(t)$ as $z(t)=z_{1}(t)+z_{2 a}(t)+z_{2 b}(t)$, where $z_{1}(t):=P_{l} z(t), z_{2 a}(t):=\left(P_{n}-P_{l}\right) z(t)$, $z_{2 b}(t):=\left(I-P_{n}\right) z(t), n>l$. Note that $z_{2 a}(t)+z_{2 b}(t)=z_{2}(t)$. Also, the space $H$ is expressed as

$$
H=\overbrace{P_{l} H}^{\operatorname{dim}=l} \oplus \underbrace{\overbrace{\left(P_{n}-P_{l}\right) H}^{\operatorname{dim}=n-l} \overbrace{\left(I-P_{n}\right) H}^{\operatorname{dim}=\infty}}_{=\left(I-P_{l}\right) H} .
$$

Then, the infinite-dimensional operators $A_{2}, B_{2}$, and $C_{2}$ are equivalently expressed as follows:

$$
A_{2}=\left[\begin{array}{cc}
A_{2 a} & 0 \\
0 & A_{2 b}
\end{array}\right], \quad B_{2}=\left[\begin{array}{c}
B_{2 a} \\
B_{2 b}
\end{array}\right], \quad C_{2}=\left[\begin{array}{ll}
C_{2 a} & C_{2 b}
\end{array}\right],
$$

where $A_{2 a}:=\left(P_{n}-P_{l}\right) A\left(P_{n}-P_{l}\right), B_{2 a}:=\left(P_{n}-P_{l}\right) B, C_{2 a}:=C\left(P_{n}-P_{l}\right), A_{2 b}:=$ $\left(I-P_{n}\right) A\left(I-P_{n}\right), B_{2 b}:=\left(I-P_{n}\right) B, C_{2 b}:=C\left(I-P_{n}\right)$. Here, note that the operators $A_{2 a}, B_{2 a}$, and $C_{2 a}$ are identified with matrices with appropriate size. Then, the operators $\mathcal{A}, \mathcal{B}$, and $\mathcal{C}$ of $[6]$ are expressed as

$$
\begin{gathered}
\mathcal{A}=\left[\begin{array}{cccc}
-A_{1}-G_{1} C_{1} & 0 & 0 & 0 \\
B_{1} F_{1} & -A_{1}-B_{1} F_{1} & 0 & 0 \\
B_{2 a} F_{1} & -B_{2 a} F_{1} & -A_{2 a} & 0 \\
B_{2 b} F_{1} & -B_{2 b} F_{1} & 0 & -A_{2 b}
\end{array}\right], \\
\mathcal{B}=\left[\begin{array}{c}
-G_{1} \\
0 \\
0 \\
0
\end{array}\right], \quad \mathcal{C}=\left[\begin{array}{llll}
0 & 0 & C_{2 a} & C_{2 b}
\end{array}\right] .
\end{gathered}
$$

Further, we set the truncated operators as

$$
\begin{gathered}
\mathcal{A}_{n}=\left[\begin{array}{ccc}
-A_{1}-G_{1} C_{1} & 0 & 0 \\
B_{1} F_{1} & -A_{1}-B_{1} F_{1} & 0 \\
B_{2 a} F_{1} & -B_{2 a} F_{1} & -A_{2 a}
\end{array}\right], \\
\mathcal{B}_{n}=\left[\begin{array}{c}
-G_{1} \\
0 \\
0
\end{array}\right], \quad \mathcal{C}_{n}=\left[\begin{array}{lll}
0 & 0 & C_{2 a}
\end{array}\right] .
\end{gathered}
$$


Now, let us define two transfer functions as follows:

$$
\begin{aligned}
& G(j \omega)=\mathcal{C}(j \omega I-\mathcal{A})^{-1} \mathcal{B}, \\
& G_{n}(j \omega)=\mathcal{C}_{n}\left(j \omega I-\mathcal{A}_{n}\right)^{-1} \mathcal{B}_{n} .
\end{aligned}
$$

The following theorem and remarks are our main result in this paper.

Theorem 2.1. Suppose that Assumption 1 is satisfied. Then, the operator $\mathcal{A}_{n}$ defined by 10 generates a $C_{0}$-semigroup $e^{t \mathcal{A}_{n}}$ with norm bound $\left\|e^{t \mathcal{A}_{n}}\right\| \leq M e^{-\nu t}, t \geq 0$ on the Euclidean space $Z_{n}:=\mathbf{R}^{l} \times \mathbf{R}^{l} \times \mathbf{R}^{n-l}$, where $M \geq 1$ and $\nu>0$ are some constants independent of the integer $n$. Moreover, there holds

$$
\left\|G_{n}(\cdot)\right\|_{\infty} \rightarrow\|G(\cdot)\|_{\infty} \quad \text { as } \quad n \rightarrow \infty
$$

that is, $r_{c}\left(\mathcal{A}_{n} ; \mathcal{B}_{n}, \mathcal{C}_{n}\right) \rightarrow r_{c}(\mathcal{A} ; \mathcal{B}, \mathcal{C})$ as $n \rightarrow \infty$. Accordingly, if $\left\|G_{n}(\cdot)\right\|_{\infty}<1$ is satisfied for sufficiently large $n$, the control law (4) works as a finite-dimensional stabilizing controller for system (1), (2).

Proof. By Assumption 1, the $C_{0}$-semigroup generated by the matrix

$$
\mathcal{A}_{1}:=\left[\begin{array}{cc}
-A_{1}-G_{1} C_{1} & 0 \\
B_{1} F_{1} & -A_{1}-B_{1} F_{1}
\end{array}\right]
$$

has a norm bound $\left\|e^{t \mathcal{A}_{1}}\right\| \leq M_{1} e^{-\nu_{1} t}, t \geq 0$, where $M_{1} \geq 1$ and $0<\nu_{1}<\lambda_{l+1}$ are some constants independent of the integer $n$. Also, the $C_{0}$-semigroup generated by the matrix $-A_{2 a}$ has a norm bound $\left\|e^{-t A_{2 a}}\right\| \leq e^{-\lambda_{l+1} t}, t \geq 0$. Here, noting that $\left\|B_{2 a} F_{1}\right\| \leq\|B\|\left\|F_{1}\right\|$, we see that the first assertion holds with $M=M_{1}\left(1+\frac{2 M_{1}\|B\|\left\|F_{1}\right\|}{\lambda_{l+1}-\nu_{1}}\right)$ and $\nu=\nu_{1}$.

Next, we estimate the $H_{\infty}$-norm of $G(j \omega)-G_{n}(j \omega)$. From $(9)-(12)$, we have

$$
\begin{aligned}
& G(j \omega)=C_{2 a}\left(j \omega I+A_{2 a}\right)^{-1} B_{2 a} H(j \omega)+C_{2 b}\left(j \omega I+A_{2 b}\right)^{-1} B_{2 b} H(j \omega), \\
& G_{n}(j \omega)=C_{2 a}\left(j \omega I+A_{2 a}\right)^{-1} B_{2 a} H(j \omega),
\end{aligned}
$$

by straightforward calculation, where

$$
H(j \omega):=-F_{1}\left(j \omega I+A_{1}+B_{1} F_{1}\right)^{-1}\left(j \omega I+A_{1}\right)\left(j \omega I+A_{1}+G_{1} C_{1}\right)^{-1} G_{1} .
$$

From these, it follows that

$$
G(j \omega)-G_{n}(j \omega)=C_{2 b}\left(j \omega I+A_{2 b}\right)^{-1} B_{2 b} H(j \omega) .
$$

By Assumption 1, it is easy to see that $\|H(\cdot)\|_{\infty}<+\infty$. Also, noting that $\left\|e^{-t A_{2 b}}\right\| \leq$ $e^{-\lambda_{n+1} t}, t \geq 0$, and that by Hille-Yosida's theorem [9, Theorem 1.5.3 and Remark 1.5.4], $\left\|\left(\lambda I+A_{2 b}\right)^{-k}\right\| \leq \frac{1}{\left(\operatorname{Re} \lambda+\lambda_{n+1}\right)^{k}}, \operatorname{Re} \lambda>-\lambda_{n+1}, k=1,2, \ldots$, we have

$$
\left\|\left(\cdot I+A_{2 b}\right)^{-1}\right\|_{\infty}=\sup _{\omega \in \mathbf{R}}\left\|\left(j \omega I+A_{2 b}\right)^{-1}\right\| \leq \frac{1}{\lambda_{n+1}} \rightarrow 0 \quad \text { as } \quad n \rightarrow \infty .
$$


Moreover noting that $\left\|B_{2 b}\right\|,\left\|C_{2 b}\right\| \rightarrow 0$ as $n \rightarrow \infty$, we have

$$
\|\| G(\cdot)\left\|_{\infty}-\right\| G_{n}(\cdot)\left\|_{\infty} \mid \leq\right\| G(\cdot)-G_{n}(\cdot)\left\|_{\infty} \leq\right\| C_{2 b}\|\|\left(\cdot I+A_{2 b}\right)^{-1}\left\|_{\infty}\right\| B_{2 b}\|\| H(\cdot) \|_{\infty} \rightarrow 0
$$

as $n \rightarrow \infty$, which implies that the second assertion holds.

From the second assertion, it follows that $\|G(\cdot)\|_{\infty}<1$ if $\left\|G_{n}(\cdot)\right\|_{\infty}<1$ for sufficiently large $n$, which implies from Theorem 1.1 that the third assertion holds.

Remark 2.2. When the output equation (8) is used instead of (2), we obtain the similar result as in Theorem 2.1. In this case, the control law (4) is replaced as

$$
\left\{\begin{array}{l}
\dot{w}_{1}(t)=-A_{1} w_{1}(t)+B_{1} u(t)+G_{1}\left(y(t)-\tilde{C}_{1}\left(A_{1}+c\right)^{\gamma} w_{1}(t)\right), \quad w_{1}(0)=w_{10} \\
u(t)=-F_{1} w_{1}(t)
\end{array}\right.
$$

as a result, the operators $C_{1}, C_{2 a}$, and $C_{2 b}$ are replaced as $\tilde{C}_{1}\left(A_{1}+c\right)^{\gamma}, \tilde{C}_{2 a}\left(A_{2 a}+c\right)^{\gamma}$, and $\tilde{C}_{2 b}\left(A_{2 b}+c\right)^{\gamma}$ in the operators $\left.(9), 10\right)$. Therefore, we need to use the following estimate instead of 130 :

$$
\left\|\left(A_{2 b}+c\right)^{\gamma}\left(\cdot I+A_{2 b}\right)^{-1}\right\|_{\infty} \leq \frac{\left(\lambda_{n+1}+c\right)^{\gamma}+\lambda_{n+1}^{\gamma} \Gamma(1-\gamma)}{\lambda_{n+1}} \rightarrow 0 \quad \text { as } \quad n \rightarrow \infty,
$$

where $\Gamma(\cdot)$ is the gamma function. For the derivation of $[15]$, see [14].

Remark 2.3. System (1), (2) is the parabolic distributed parameter system which contains a diffusion process and a transport-diffusion process, and so on. The assertions of Theorem 2.1 also hold in the case where the operator $A$ is replaced by a Riesz-spectral operator, that is, for the system described by a flexible beam equation.

Remark 2.4. In Theorem 2.1, one cannot give a priori estimate with respect to $n$ for assuring $\left\|G_{n}(\cdot)\right\|_{\infty}<1$.

Remark 2.5. According to the procedure in [5], we wrote the closed-loop system consisting of system (1), (2) and the controller (4) as equation (5). But, we may consider the other expression such as

$$
\dot{\xi}(t)=\left(\mathcal{A}^{\prime}+\mathcal{B}^{\prime} \mathcal{K}^{\prime} \mathcal{C}^{\prime}\right) \xi(t), \quad \xi(0)=\xi_{0},
$$

where the operators $\mathcal{A}^{\prime}, \mathcal{B}^{\prime}, \mathcal{C}^{\prime}$, and $\mathcal{K}^{\prime}$ are defined by

$$
\begin{gathered}
\mathcal{A}^{\prime}=\left[\begin{array}{cccc}
-A_{1}-G_{1} C_{1} & 0 & -G_{1} C_{2 a} & -G_{1} C_{2 b} \\
B_{1} F_{1} & -A_{1}-B_{1} F_{1} & 0 & 0 \\
0 & 0 & -A_{2 a} & 0 \\
0 & 0 & 0 & -A_{2 b}
\end{array}\right], \\
\mathcal{B}^{\prime}=\left[\begin{array}{c}
0 \\
0 \\
B_{2 a} \\
B_{2 b}
\end{array}\right], \quad \mathcal{C}^{\prime}=\left[\begin{array}{llll}
F_{1} & -F_{1} & 0 & 0
\end{array}\right], \quad \mathcal{K}^{\prime}=1 .
\end{gathered}
$$


Based on this expression, we can obtain the similar results as in Theorems 1.1 and 2.1. Especially, in the case of single input and single output system, noting that the transfer function $G^{\prime}(j \omega):=\mathcal{C}^{\prime}\left(j \omega I-\mathcal{A}^{\prime}\right)^{-1} \mathcal{B}^{\prime}$ is equal to the transfer function $G(j \omega)$ defined by (11), we have the same stability radius for the closed-loop systems (5) and (16), i. e., $r_{c}\left(\mathcal{A}^{\prime} ; \mathcal{B}^{\prime}, \mathcal{C}^{\prime}\right)=r_{c}(\mathcal{A} ; \mathcal{B}, \mathcal{C})$. As a result, we see that $r_{c}\left(\mathcal{A}_{n}^{\prime} ; \mathcal{B}_{n}^{\prime}, \mathcal{C}_{n}^{\prime}\right)=r_{c}\left(\mathcal{A}_{n} ; \mathcal{B}_{n}, \mathcal{C}_{n}\right)$ holds for approximate operators.

\section{NUMERICAL EXAMPLE}

We consider the following parabolic distributed parameter system:

$$
\left\{\begin{array}{l}
z_{t}(t, x)=\varepsilon z_{x x}(t, x)+\alpha z_{x}(t, x)+\mu z(t, x)+b(x) u(t), \quad t>0, x \in(0,1), \\
z_{x}(t, 0)=0, \quad z(t, 1)=0, \quad t>0 \\
z(0, x)=z_{0}(x), \quad x \in[0,1]
\end{array}\right.
$$

where $z(t, x) \in \mathbf{R}$ is the temperature at time $t$ and at the point $x \in[0,1], u(t) \in$ $\mathbf{R}$ is the control input, and, $\varepsilon>0$ and $\alpha, \mu \geq 0$ are physical parameters. $b(x):=$ $\frac{1}{r} \mathbf{1}_{\left[x_{0}-r / 2, x_{0}+r / 2\right]}(x)$ denotes the actuator influence function, where $\mathbf{1}_{[\cdot, \cdot]}(x)$ denotes the characteristic function. We first consider the following observation for system (18):

$$
y(t)=\int_{0}^{1} c(x) z(t, x) \mathrm{d} x,
$$

where $c(x):=\frac{1}{r} \mathbf{1}_{\left[x_{1}-r / 2, x_{1}+r / 2\right]}(x)$ is the sensor influence function.

Let $\beta:=\frac{\alpha}{\varepsilon}$. We formulate system $18,{ }_{19}$ in a Hilbert space $L_{\beta}^{2}(0,1)$, where $L_{\beta}^{2}(0,1)$ is the weighted $L^{2}$-space with inner product

$$
\langle\varphi, \psi\rangle_{\beta}:=\int_{0}^{1} \varphi(x) \psi(x) e^{\beta x} \mathrm{~d} x, \quad \varphi, \psi \in L_{\beta}^{2}(0,1) .
$$

Setting $\mathcal{L} \varphi=-\varepsilon \varphi^{\prime \prime}-\alpha \varphi^{\prime}-\mu \varphi$, we define the unbounded operator $A: D(A) \subset L_{\beta}^{2}(0,1) \rightarrow$ $L_{\beta}^{2}(0,1)$ as

$$
\begin{aligned}
& A \varphi=\mathcal{L} \varphi, \quad \varphi \in D(A) \\
& D(A)=\left\{\varphi \in H^{2}(0,1) ; \varphi^{\prime}(0)=0, \varphi(1)=0\right\} .
\end{aligned}
$$

Then, $A$ is a self-adjoint operator in $L_{\beta}^{2}(0,1)$ and it has the following eigenvalues and eigenfunctions:

$$
\begin{aligned}
& \lambda_{i}=\omega_{i}^{2} \varepsilon+\frac{\alpha^{2}}{4 \varepsilon}-\mu, \\
& \varphi_{i}(x)=\left(\frac{1}{2}+\frac{\varepsilon}{\alpha} \cos ^{2} \omega_{i}\right)^{-\frac{1}{2}} e^{-\frac{\alpha}{2 \varepsilon} x} \sin \omega_{i}(1-x),
\end{aligned}
$$

$i \geq 1$, where $\omega_{1}<\omega_{2}<\cdots<\omega_{i}<\cdots$ are the solutions of $\tan \omega=-\frac{2 \varepsilon}{\alpha} \omega$ on $\omega>0$, and $\left\{\varphi_{i}\right\}_{i=1}^{\infty}$ forms a complete orthogonal system in $L_{\beta}^{2}(0,1)$. Note that the operator $-A$ 
generates an analytic semigroup $e^{-t A}$ on $L_{\beta}^{2}(0,1)$ whose growth bound is equal to $-\lambda_{1}$. If $-\lambda_{1}>0$, it is clear that system 18,19 is unstable. Here, by defining the bounded operators $B: \mathbf{R} \rightarrow L_{\beta}^{2}(0,1)$ and $C: L_{\beta}^{2}(0,1) \rightarrow \mathbf{R}$ as

$$
\begin{aligned}
& B v=b v, \quad v \in \mathbf{R}, \\
& C \zeta=\left\langle e^{-\beta \cdot} c, \zeta\right\rangle_{\beta}, \quad \zeta \in L_{\beta}^{2}(0,1),
\end{aligned}
$$

system (18), 19) is expressed as in (1), 2).

Next, we consider the following boundary observation for system (18):

$$
y(t)=z_{x}(t, 1) .
$$

In this case, we can formulate the observation equation 20 as

$$
y(t)=\tilde{C}(A+c)^{\gamma} z(t),
$$

where $\gamma:=\frac{3}{4}+\epsilon^{\prime} \in\left(\frac{3}{4}, 1\right)$, and $\tilde{C}: L_{\beta}^{2}(0,1) \rightarrow \mathbf{R}$ is the bounded operator defined by

$$
\tilde{C} \xi=\left\langle-\frac{1}{\varepsilon}(A+c)^{\frac{1}{4}-\epsilon^{\prime}} h, \xi\right\rangle_{\beta}, \quad \xi \in L_{\beta}^{2}(0,1) .
$$

In the above, $h \in H^{2}(0,1)$ is the unique solution of the boundary value problem

$$
(\mathcal{L}+c) h=0 \text { in }(0,1), \quad h^{\prime}(0)=0, \quad h(1)=e^{-\beta} .
$$

Especially, when $c=\mu$, the solution is concretely given by $h(x)=e^{-\beta}$. For the derivation of (21), see e.g. [15].

Now, let $\varepsilon=0.1, \alpha=0, \mu=1, x_{0}=0.8, x_{1}=0.4, r=0.02$, and $\epsilon^{\prime}=0.15$. Then, the eigenvalues and eigenfunctions of the operator $A$ become $\lambda_{i}=0.1\left(i-\frac{1}{2}\right)^{2} \pi^{2}-1, \varphi_{i}(x)=$ $\sqrt{2} \cos \left(i-\frac{1}{2}\right) \pi x, i \geq 1$. As a result, we see that $-A$ has one unstable eigenvalue. Next, by setting $l=3$, we can derive two models $\left(-A_{1}, B_{1}, C_{1}\right)$ and $\left(-A_{1}, B_{1}, \tilde{C}_{1}\left(A_{1}+\mu\right)^{\gamma}\right)$ that satisfy Assumption 1. In fact, these models are controllable and observable [17, and they correspond to the low order finite-dimensional models of system $(18),(19)$ and system (18), 20). For each model, let us choose $F_{1}$ as an optimal regulator gain [17] and choose $G_{1}$ as an optimal filter gain [17, with the weights $Q=2 I_{3}$ and $R=1$. Then, for the model $\left(-A_{1}, B_{1}, C_{1}\right)$, we have

$$
\begin{aligned}
& F_{1}=\left[\begin{array}{lll}
5.3149 & -0.1541 & 0.1733
\end{array}\right], \quad G_{1}=\left[\begin{array}{r}
2.3165 \\
-0.0533 \\
-0.1680
\end{array}\right], \\
& \sigma\left(-A_{1}-B_{1} F_{1}\right)=\{-0.8102,-1.9880,-5.5814\}, \\
& \sigma\left(-A_{1}-G_{1} C_{1}\right)=\{-1.1507,-1.8157,-5.5804\},
\end{aligned}
$$

where $\sigma\left(-A_{1}-B_{1} F_{1}\right)$ denotes the set of eigenvalues of the matrix $-A_{1}-B_{1} F_{1}$. Similarly, for the model $\left(-A_{1}, B_{1}, \tilde{C}_{1}\left(A_{1}+\mu\right)^{\gamma}\right)$, we have

$$
\begin{aligned}
& F_{1}=\left[\begin{array}{lll}
5.3149 & -0.1541 & 0.1733
\end{array}\right], \quad G_{1}=\left[\begin{array}{r}
-4.3337 \\
0.2356 \\
-0.5369
\end{array}\right], \\
& \sigma\left(-A_{1}-B_{1} F_{1}\right)=\{-0.8102,-1.9880,-5.5814\}, \\
& \sigma\left(-A_{1}-G_{1} \tilde{C}_{1}\left(A_{1}+\mu\right)^{\gamma}\right)=\{-0.8075,-2.8760,-19.1124\} .
\end{aligned}
$$


For the both cases, we use the same notation $G_{n}$ to indicate the transfer functions of approximate feedback control systems. Figures 1 and 2 show that the value of $\left\|G_{n}(\cdot)\right\|_{\infty}$ converges to some value less than 1 as $n$ goes to infinity, which means from Theorem 2.1 that the control law (4) (resp. the control law (14)) works as a finite-dimensional stabilizing controller for system (18), (19) (resp. system (18), (20)), by making choice of such $l, F_{1}$, and $G_{1}$. Here, note that the convergence speed of the case with boundary observation (20) is late compared with that of the case with distributed observation (19).

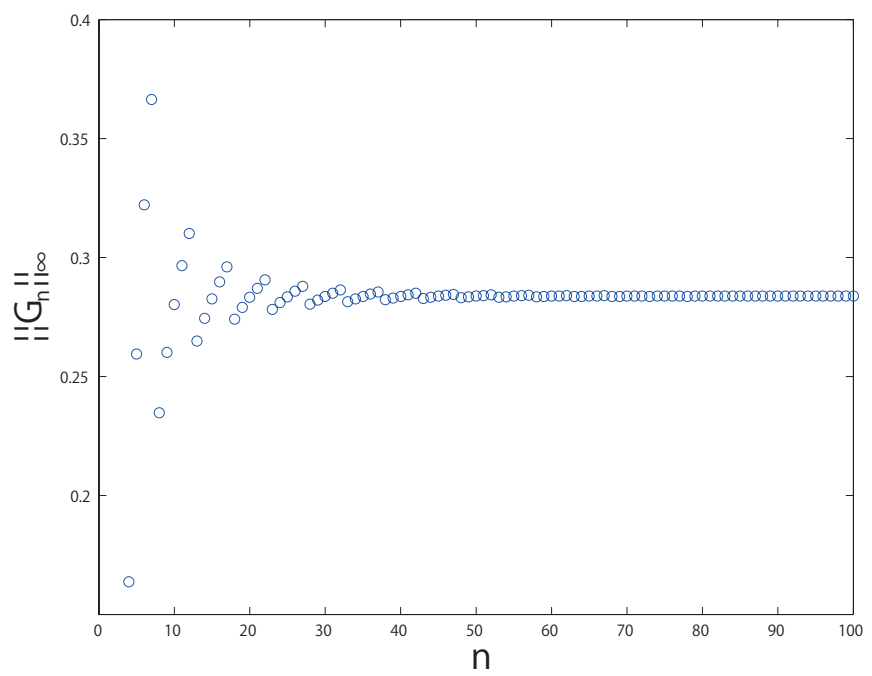

Fig. 1. The case of distributed observation 19$) .\left\|G_{100}\right\|_{\infty}=0.2838(<1)$.

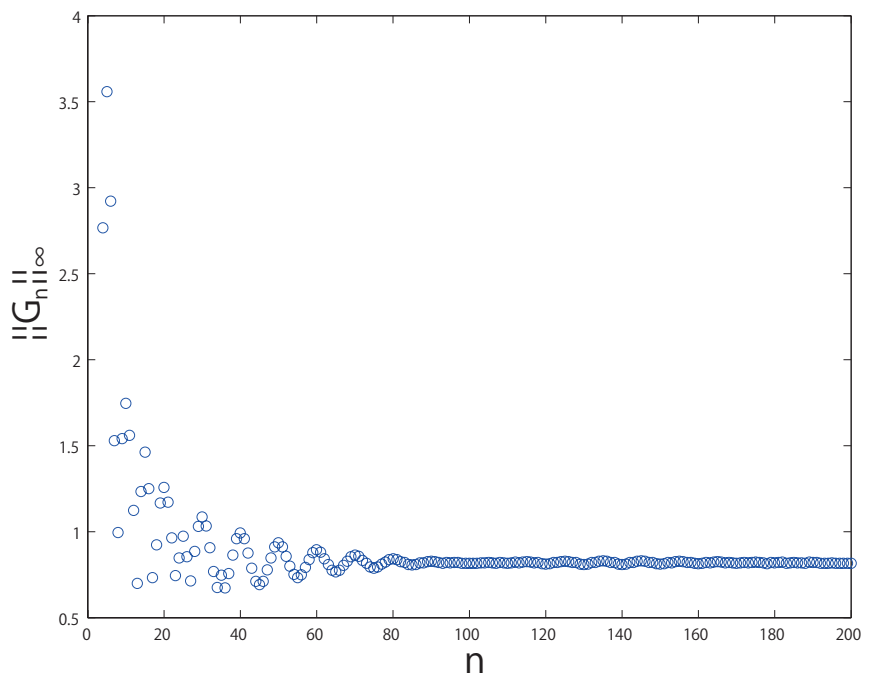

Fig. 2. The case of boundary observation $(20) .\left\|G_{200}\right\|_{\infty}=0.8160(<1)$. 
The difference is caused by the estimates $\sqrt{13}$ and $\sqrt{15}$. In the numerical simulation, we used MATLAB Control System Toolbox.

\section{CONCLUSIONS}

In this paper, in connection with the work of [5], we studied the problem of approximating stability radius appearing in the design of finite-dimensional stabilizing controllers for an infinite-dimensional dynamical system. From the computational point of view, we needed to prepare a family of approximate finite-dimensional operators and then to calculate the $H_{\infty}$-norm of their transfer functions. Theorem 2.1 assures that they converge to the value of $H_{\infty}$-norm of the original transfer function. In the future, we plan to study the similar problem for the case where the system operator is not expressed by a Riesz-spectral operator as well as for the case with input delay.

\section{ACKNOWLEDGEMENTS}

The author would like to thank the anonymous reviewers for their valuable comments. This research is partially supported by KAKENHI (Grant-in-Aid for Scientific Research (C), No. 15K04999), Japan Society for the Promotion of Science.

(Received February 18, 2016)

\section{REFERENCES}

[1] M. J. Balas: Finite-dimensional controllers for linear distributed parameter systems: exponential stability using residual mode filters. J. Math. Anal. Appl. 133 (1988), 283296. DOI:10.1016/0022-247x(88)90401-5

[2] C. Chicone and Y. Latushkin: Evolution Semigroups in Dynamical Systems and Differential Equations. Mathematical Surveys and Monographs, vol. 70. American Mathematical Society, 1999. DOI:10.1090/surv/070

[3] R.F. Curtain: Finite dimensional compensators for parabolic distributed systems with unbounded control and observation. SIAM J. Control Optim. 22 (1984), 255-276. DOI:10.1137/0322018

[4] R. Glowinski, J.-L. Lions, and J. He: Exact and Approximate Controllability for Distributed Parameter Systems: A Numerical Approach. In: Encyclopedia of Mathematics and Its Applications, vol. 117. Cambridge University Press, Cambridge 2008.

[5] A. El Jai and A. J. Pritchard: Sensors and Controls in the Analysis of Distributed Systems. English Language Edition. Ellis Horwood Limited, Chichester 1988.

[6] N. Guglielmi and M. Manetta: Approximating real stability radii. IMA J. Numerical Analysis 35 (2015), 1402-1425. DOI:10.1093/imanum/dru038

[7] I. Lasiecka and R. Triggiani: Control Theory for Partial Differential Equations: Continuous and Approximation Theories I: Abstract Parabolic Systems. In: Encyclopedia of Mathematics and Its Applications, vol. 74. Cambridge University Press, Cambridge 2000. DOI:10.1017/cbo9780511574801

[8] T. Nambu: On stabilization of partial differential equations of parabolic type: boundary observation and feedback. Funkcialaj Ekvacioj, Serio Internacia 28 (1985), 267-298. 
[9] A. Pazy: Semigroups of Linear Operators and Applications to Partial Differential Equations. In: Applied Mathematical Sciences, vol. 44. Springer-Verlag, New York 1983. DOI:10.1007/978-1-4612-5561-1

[10] A. J. Pritchard and S. Townley: Robust compensator design via structured stability radii. Systems Control Lett. 11 (1988), 33-37. DOI:10.1016/0167-6911(88)90108-9

[11] A. J. Pritchard and S. Townley: Robustness of linear systems. J. Differential Equations 77 (1989), 254-286. DOI:10.1016/0022-0396(89)90144-7

[12] Y. Sakawa: Feedback stabilization of linear diffusion systems. SIAM J. Control Optim. 21 (1983), 667-676. DOI:10.1137/0321040

[13] H. Sano and N. Kunimatsu: Feedback stabilization of infinite-dimensional systems with $A^{\gamma}$-bounded output operators. Appl. Math. Lett. 7 (1994), 5, 17-22. DOI:10.1016/08939659(94)90065-5

[14] H. Sano: Finite-dimensional $H_{\infty}$ control of linear parabolic systems with unbounded output operators. Int. J. Control 72 (1999), 16, 1466-1479. DOI:10.1080/002071799220119

[15] H. Sano: Stability-enhancing control of a coupled transport-diffusion system with Dirichlet actuation and Dirichlet measurement. J. Math. Anal. Appl. 388 (2012), 1194-1204. DOI:10.1016/j.jmaa.2011.11.011

[16] J. M. Schumacher: A direct approach to compensator design for distributed parameter systems. SIAM J. Control Optim. 21 (1983), 823-836. DOI:10.1137/0321050

[17] K. Zhou, J. Doyle, and K. Glover: Robust and Optimal Control. (In Japanese, translated from the English by K. Z. Liu and Z. H. Luo.) Corona, Tokyo 1997.

Hideki Sano, Department of Applied Mathematics, Graduate School of System Informatics, Kobe University, 1-1 Rokkodai, Nada, Kobe 657-8501. Japan.

e-mail:sano@crystal.kobe-u.ac.jp 